\title{
TECNOLOGIAS EDUCATIVAS FACILITADORAS NA PROMOÇÃO DA SAÚDE PROJETO DE EXTENSÃO "BATE PAPO: SAÚDE NA ESCOLA": RELATO DE EXPERIÊNCIA
}

\author{
FACILITATIVE EDUCATIONAL TECHNOLOGIES IN HEALTH \\ PROMOTION. EXTENSION PROJECT "CHAT: HEALTH AT SCHOOL": \\ EXPERIENCE REPORT
}

\author{
Jásny Pintor de Assis Correia ${ }^{1}$ \\ Guilherme Vieira Lima \\ José Vinícius de Souza ${ }^{3}$ \\ Ruan Souza Alixandre ${ }^{4}$ \\ Marília Andreza da Silva Ferreira ${ }^{5}$ \\ Flávio Lourenço de Oliveira ${ }^{6}$
}

RESUMO: INTRODUÇÃO: o ambiente escolar pode ser utilizado/caracterizado como um eficiente meio para viabilizar ações que visem promoção da saúde, oportunizando interações que facilitam o diagnóstico e a prevenção de doenças anteriormente à adoção de comportamentos de risco. Para tanto, o uso tecnologias educacionais surge como uma importante ferramenta na construção do conhecimento, sendo capaz de consolidar também, o processo de ensino e aprendizagem. OBJETIVOS: relatar a experiência do uso de métodos ativos do aprendizado na execução de ações educativas em saúde, relacionadas a temas de grande relevância na atualidade, em uma escola da Rede Estadual de Ensino pelo

\footnotetext{
${ }^{1}$ Acadêmica do Curso de Medicina da Universidade Federal de Campina Grande, Paraíba, Brasil. Email: jasnypintor10@gmail.com.

${ }^{2}$ Acadêmico do Curso de Medicina da Universidade Federal de Campina Grande, Paraíba, Brasil. Email: guiga_sjp@hotmail.com.

${ }^{3}$ Acadêmico do Curso de Medicina da Universidade Federal de Campina Grande, Paraíba, Brasil. Email: vinicius.souza15@hotmail.com.

${ }^{4}$ Acadêmico do Curso de Medicina da Universidade Federal de Campina Grande, Paraíba, Brasil. Email: ruansouzaalixandre@gmail.com.

${ }^{5}$ Técnico de Laboratório/ Análises Clínicas/ CFP/ UFCG; Mestre em Ciências Biológicas/UFPE. Email: marilia.andreza.masf@gmail.com.

${ }^{6}$ Doutorando em Recursos Naturais pelo Programa de Pós-graduação em Recursos Naturais da Universidade Federal de Campina Grande (UFCG). Biólogo e Advogado - Téc. do Laboratório de Microbiologia do Centro de Formação de Professores da Universidade Federal de Campina Grande (UFCG). E-mail: flaviolourenco_3@hotmail.com.
} 
projeto "Bate Papo: Saúde na Escola". MÉTODOS: trata-se de um estudo descritivo, do tipo relato de experiência, com base no Projeto de Extensão "Bate Papo: Saúde na Escola". As ações foram respaldadas na realização de rodas de conversa, utilização de dispositivos para aplicação de jogos, além de oficinas práticas fundamentadas na demonstração de exames e métodos contraceptivos. Foram abordadas algumas Infecções Sexualmente Transmissíveis (ISTs), arboviroses e doenças fúngicas. As atividades foram desenvolvidas, sob a supervisão do Coordenador e da equipe de Orientadores, por alunos de Graduação dos cursos de Enfermagem e Medicina, da Universidade Federal de Campina Grande, Campus Cajazeiras/PB, na Escola Estadual Professor Manoel Mangueira Lima, ligada à $9^{a}$ Gerência Regional de Ensino, desta cidade, beneficiando, aproximadamente, 150 alunos. RESULTADOS: a proposta superou as expectativas em função da receptividade do público alvo às metodologias alternativas de ensino e aprendizagem, bem como a construção conjunta de saberes. Foi oportunizado aos profissionais de saúde, em formação, o contato com a comunidade, para a troca de experiências e conhecimento de sua realidade e seus anseios, que, de forma recíproca, recebeu dos extensionistas, intervenções facilitadoras à promoção da saúde. CONCLUSÃO: a utilização de metodologias alternativas, pelo projeto, mostrou-se positiva, por despertar o interesse e a motivação no aprendizado das temáticas, colaborando na construção do conhecimento do público-alvo. Ressalta-se a carência de ações promotoras de saúde na educação da rede pública de ensino e de estratégias que visem a integração familiar para discussão de temas importantes na saúde pública.

Palavras chave: Educação em Saúde, Tecnologia Educacional, Assistência à Saúde.

ABSTRACT: INTRODUCTION: the school environment can be used as an efficient means to enable actions aimed at promoting health, providing opportunities for interactions that facilitate the diagnosis and prevention of diseases before the establishment of risk behaviors. For this, the use of educational technologies emerges as an important tool in the construction of knowledge, being able to also consolidate the teaching-learning process. OBJECTIVE: to report an experience of using methods of carrying out health education actions, related to topics of current relevance, in a school of the State Education Network by the project "Chat: Health at School". METHODOLOGY: this is a descriptive study of the experience report type, based on the Extension Project "Chat: Health at School". The actions were supported by holding conversation circles, using devices to apply games, in addition to practical workshops based on demonstration of exams and contraceptive methods, so that some Sexually Transmitted Infections (STIs), arboviruses and fungal diseases were addressed. Activities were developed, under the supervision of the Coordinator and the team of Advisors, by undergraduate students of Nursing and Medicine courses, from the Federal University of Campina Grande, campus Cajazeiras / PB, at the ESCOLA ESTADUAL PROFESSOR MANOEL MANGUEIRA LIMA, linked to the 9th Regional Teaching Management of this city, benefiting approximately 150 people. RESULTS: the proposal exceeded expectations due to the receptivity of the target audience to alternative teaching and learning methodologies, as well as the joint 
construction of knowledge. Health professionals, in training, were given contact with the community to exchange experiences and knowledge of their reality and desires, which, in a reciprocal manner, received interventions that facilitate health promotion from extension workers. CONCLUSION: the use of alternative methodologies by the project proved to be positive because it aroused interest and motivation in learning the themes, collaborating in the construction of the knowledge of the target audience. The lack of health-promoting actions in public school education and strategies aimed at family integration to discuss important issues in public health are highlighted.

Keywords: Health Education, Educational Technology, Delivery of Health Care. 


\section{INTRODUÇÃO}

A definição de promoção em saúde, segundo a Carta de Ottawa, documento apresentado na Primeira Conferência Internacional sobre Promoção da Saúde (1986), refere-se ao processo de capacitação da comunidade, visando a atuação para a melhoria da qualidade de vida e saúde, o que abrange uma maior participação no controle deste processo; de forma que seja possível atingir um estado pleno de bem-estar físico, mental e social. A promoção em saúde propicia o desenvolvimento, ao nível de indivíduo e de sociedade, por meio da transmissão de conhecimento, educação para a saúde e intensificação das habilidades vitais; além de ser eficaz, ao possibilitar alterações nos determinantes da saúde, ou seja, nos fatores sob o domínio do indivíduo, dos estilos de vida e condutas; ou nos fatores externos, relacionados às condições socioeconômicas, ambientais e à prestação de serviços (WHO; 1998).

A escola configura-se como um espaço de possibilidades para promover a saúde e prevenir doenças antes do estabelecimento de comportamentos de risco, por parte dos jovens. Esta instituição formadora fornece o auxílio no estabelecimento de condutas e hábitos saudáveis, os quais podem perdurar por toda a vida adulta do indivíduo (SCHALET; 2014). O estudo de Scull et al. (2020), evidenciou estes benefícios, em virtude da demonstração de que os programas abrangentes de educação em saúde sexual, com base em evidências, são responsáveis por contribuir com a redução do início precoce da vida sexual, dos comportamentos sexuais de risco, da gravidez na fase de adolescência, das infecções sexualmente transmissíveis (ISTs), da quantidade de parceiros sexuais durante a vida, de forma a promover a educação sexual e reprodutiva dos indivíduos. Ademais, os crescentes problemas de saúde pública, tais como as arboviroses; em decorrência, por exemplo, do quantitativo de casos graves, do potencial de dispersão e da capacidade de adaptação dos vetores, exigem o estabelecimento de políticas de enfrentamento e intervenções de amplo espectro, que envolvam, não apenas os 
setores da área da saúde, mas, os diversos setores da sociedade, sobretudo, a escola (DONALISIO; FREITAS; ZUBEN, 2016). A instituição educacional possui um papel importante na construção da cidadania, devido ao fato de possibilitar a apropriação, a compreensão e o agir no mundo, por parte dos sujeitos.

Muitos projetos de promoção da saúde são centrados na regulação da vivência em sociedade e na instauração de medidas, responsáveis por delimitar o sentido do viver de forma saudável, em decorrência de dificuldades em desvencilharse da tendência behaviorista (comportamentalista). Dessa maneira, as ações de promoção da saúde, focadas no comportamento individual, acabam por responsabilizar o sujeito pela adoção de comportamentos de risco e pelo o desenvolvimento de doenças, bem como pela incorporação de hábitos e estilos de vida saudáveis (FERREIRA; CASTIEL; CARDOSO, 2017). No entanto, ressalta-se o risco, sobretudo, dos profissionais de saúde, em desrespeitar a autonomia e o modo de pensar do sujeito, a partir da imposição de um estilo de vida e de determinados comportamentos. Deste modo, é imprescindível, não somente no campo da saúde, o respeito à autonomia do outro, ou seja, à possibilidade do agir de forma autônoma, assumindo o papel de responsável por sua própria vida (BEZERRA et al.; 2013). Este princípio ético fundamental encontra-se relacionado à compreensão do direito do outro, em possuir uma forma de pensar, realizar escolhas e, de acordo com crenças e valores próprios, orientar suas ações.

Segundo Paulo Freire (2019, p. 24), “[...] ensinar não é transferir conhecimentos, mas criar as possibilidades para a sua própria produção ou a sua construção". Nesse contexto, em consonância com as ideias defendidas pelo educador e filósofo brasileiro, a educação em saúde não deve se pautar na transferência de conhecimentos sobre práticas adequadas em saúde, hábitos de vida saudáveis e prevenção de doenças, por exemplo; mas, sim, criar oportunidades para a sua própria elaboração. Em virtude desse argumento, o diálogo deve estar presente nestes momentos educativos, como um recurso relacional, por meio da aproximação entre educadores e educandos, assim como através da consciência sobre a responsabilidade dos sujeitos e de suas ações, em decorrência dos papéis desempenhados; para que seja possível o entendimento sobre o objeto de estudo, de forma a expandir, com a inserção criativa e transformadora, a prática da 
libertação (ALMEIDA; MOUTINHO; LEITE, 2016). Para tanto, as aulas práticas e as metodologias alternativas surgem como objetos de interesse, por parte dos alunos, constituindo-se como ferramentas importantes no processo de conscientização e sensibilização do grupo de estudo (SANTOS et al.; 2017).

As metodologias alternativas oportunizam o rompimento com o estilo de educação bancária, em que o sujeito permanece apenas em uma condição de passividade, recebendo o conhecimento por parte dos indivíduos que, em tese, detêm maiores níveis de saberes; para considerar os estudantes secundaristas como portadores de conhecimento prévios, de forma a proporcionar uma troca de saberes e os seus papéis como agentes ativos no processo de ensino e aprendizagem. A ludicidade, vista como uma necessidade do ser humano, independentemente da idade, é incorporada nestas tecnologias de ensino, de forma a trazer benefícios, tais como evidencia Moura et al. (2017) em seu estudo: a facilidade na aprendizagem e nos processos de socialização, na comunicação, na construção do conhecimento, da motivação pessoal e das representações mentais.

Neste sentido, o presente estudo busca elucidar sobre o uso de metodologias ativas, durante a vigência do projeto de extensão "Bate papo: saúde na escola", por meio do relato acerca da execução das ações educativas em saúde, relacionadas aos temas de relevância, na atualidade, para jovens e adolescentes, em uma escola da Rede Estadual de Ensino no alto sertão paraibano.

\section{MÉTODO}

Trata-se de um estudo descritivo, do tipo "relato de experiência", elaborado a partir da execução do projeto de extensão Bate-papo: saúde na escola, na Escola Estadual Professor Manoel Mangueira Lima (EEPMML), ligada à $9^{a}$ Gerência Regional de Ensino, em Cajazeiras/PB, e na Universidade Federal de Campina Grande (UFCG), Centro de Formação de Professores (CFP), campus situado no mesmo município. 
As atividades foram realizadas no período de maio a dezembro de 2019 , beneficiando cerca de 150 alunos, regularmente matriculados no ensino médio. As ações consistiram na capacitação dos discentes de graduação em Enfermagem e Medicina, do CFP/UFCG e, posteriormente, na realização de visitas à escola parceira, para efetuação de rodas de conversa e vivência de dinâmicas interativas sobre as temáticas: ISTS, arboviroses e doenças fúngicas, além da condução do público-alvo à visita técnica no Laboratório de Microbiologia, Parasitologia e Patologia da UFCG (LMPP-UFCG).

As ações foram respaldadas nas apresentações expositiva e dialogada dos temas propostos, com a subsequente aplicação de Tecnologias Educativas (TE). Foram utilizados o "Jogo Torre da Saúde", cedido pela parceria com o Laboratório de Tecnologias de Informação e Comunicação em Saúde (LATICS), e o "Jogo do Repolho"; além da demonstração do exame pélvico feminino, em manequim, da exposição e explicação de métodos contraceptivos, durante a visita às instalações da Universidade.

A avaliação do presente estudo, pelo Comitê de Ética, fez-se desnecessária, devido ao estudo tratar-se de um relato de experiência. Ressalta-se o cumprimento da produção de conteúdo de maneira ética, considerando-se a preservação do anonimato dos participantes, de acordo com a resolução 466/12, aprovada pelo Conselho Nacional de Saúde.

As experiências descritas dizem respeito às vivências dos extensionistas, nas ações desenvolvidas nos dias 22 de Outubro de 2019 e 21 de Novembro de 2019, na UFCG e EEPMML, respectivamente.

\section{RELATO DE EXPERIÊNCIA}

As práticas realizadas por meio do Projeto de Extensão buscaram expandir os conhecimentos adquiridos na Universidade, até os espaços do Ensino Médio público estadual. Para essa intervenção, mecanismos de promoção à saúde, com dinâmicas 
que abordassem as IST's, doenças fúngicas e arboviroses, foram criados pela equipe atuante.

\section{Ação no Laboratório de Microbiologia, Parasitologia e Patologia - UFCG}

No dia 22 de Outubro de 2019, a equipe de extensionistas conduziu os estudantes da escola contemplada, para a realização das atividades nos Laboratórios de Microbiologia, Parasitologia e Patologia da UFCG. Inicialmente, foram debatidas as principais ISTs, na forma de roda de conversa. Dessa maneira, adequaram-se determinados termos científicos a uma linguagem melhor compreendida pelos estudantes, relacionando-os às denominações populares; ao mesmo tempo em que houve a preocupação em desconstruir palavras usadas erroneamente. Dentre os patógenos, o que mais gerou curiosidade entre os alunos, foi o vírus HIV, percebido como tema de grandes controvérsias, preconceitos, bem como de muitos mitos, quanto às suas formas de contágio e tratamento. A Sífilis também foi uma IST que causou significativo debate, pela preocupante situação dessa infecção no Brasil.

Em um segundo momento, a equipe trabalhou com a "Torre da Saúde", tecnologia educativa do acervo do LATICS. O jogo é composto por peças numeradas e empilhadas; e uma caixa contendo bolas numeradas correspondentes a cada peça que compõe a torre. Os estudantes são divididos em duas equipes; e perguntas, a respeito do tema trabalhado, são realizadas a cada uma. Se a equipe questionada errar a resposta, deverá sortear uma bola da caixa e retirar a peça da torre, que possui o número sorteado, de maneira que, a torre não caia. Se acertar, a equipe adversária terá de realizar o sorteio e retirar o bloco, sem desmoronar a construção. Perde o jogo, a equipe que derrubar a torre. As perguntas, aliadas ao aspecto lúdico do método, foram imprescindíveis para criar um ambiente de desinibição e desconstrução de mitos. Diante dos assuntos mais abordados na brincadeira: prevenção, formas de transmissão, tratamento e cuidados íntimos no contexto das ISTs, foi observado um maior interesse das equipes, em relação às 
conversas; e uma compreensão mais clara e rápida sobre o conteúdo trabalhado/debatido.

$\mathrm{Na}$ última parte da abordagem, a ação contou com o uso de peças anatômicas representando os órgãos sexuais masculino e feminino, para demonstração de diferentes procedimentos inerentes ao tema; bem como foi realizada, também, a demonstração de métodos contraceptivos; dentre eles, o uso correto do preservativo. É importante destacar que, ao se perguntar aos estudantes, antes da exposição, sobre o uso do preservativo feminino e sobre a realização do exame citológico, percebeu-se o seu desconhecimento e o preconceito, entre eles, sobre estes métodos de prevenção, de tal maneira que foi revelada a escassez de orientação sobre tais assuntos, tanto pelas famílias, pelas escolas, como também, pelos órgãos de saúde.

Ressalta-se que o preservativo masculino era conhecido pelos estudantes, embora a sua utilização fosse vinculada apenas à contracepção. Fez-se, ainda, considerações sobre o seu uso correto e sobre a ideia equivocada de vincular a mulher à condição de responsável, pela exigência do parceiro, ao uso do preservativo, durante a relação sexual, de forma a desconsiderar a responsabilidade mútua da prevenção. Foi discutida, ainda, a errônea dissociação da necessidade de seu uso em relações, entre pessoas do mesmo sexo. A exposição prática desmistificou a realização de exames pélvicos de rotina, procedimentos esses que eram desconhecidos por muitos dos alunos; ajudando, assim, a reduzir o estigma e os possíveis medos que interferem na busca ativa por profissionais da saúde, garantindo a segurança desses jovens, em experiências futuras.

\section{Ação na Escola Estadual Professor Manoel Mangueira Lima}

A visita realizada à escola parceira, no dia 21 de Novembro de 2019, ocorreu com a formação de uma roda de conversa, entre os alunos e os extensionistas, utilizando apresentação de slides, estrategicamente construídos com figuras, mapas mentais e quadros didáticos, de forma a facilitar a compreensão sobre as temáticas 
trabalhadas. A equipe do projeto dividiu-se, de modo que, cada membro moderou a discussão a respeito de duas patologias pertinentes. Foram explorados, na ação, temas relevantes no contexto epidemiológico da região, como arboviroses e doenças fúngicas.

A cada tema discutido, os alunos realizavam perguntas a respeito do assunto, revelando interesse acerca do conteúdo. Na discussão, a equipe notou deficiências no entendimento de conteúdos básicos de Biologia, a partir das perguntas e discussões realizadas pelos adolescentes e jovens.

Posteriormente, utilizou-se a dinâmica denominada pela equipe, de "Jogo do Repolho". A brincadeira consistia na produção de uma bola de papel, simulando um repolho, em que suas camadas são feitas de folhas amassadas, de forma que, em cada folha, foi escrita uma pergunta sobre os assuntos recém discutidos, ou a proposta de uma "prenda", como estímulo à diversão. Os estudantes foram dispostos em uma conformação circular, de modo que, a cada rodada, repassavam o repolho, ao som de uma música reproduzida pela equipe. Ao ser interrompida a música, o participante que estivesse com o repolho em mãos, retirava a camada mais superficial da bola de papel, devendo responder à pergunta ou "pagar a prenda".

A equipe sentiu-se satisfeita com a contribuição da dinâmica, para a motivação no aprendizado das temáticas e para a construção do conhecimento, em virtude da aceitação e participação na dinâmica, por parte dos alunos. Inclusive, foi gerada uma disputa, no grupo, para responder às perguntas, quando algum colega não sabia a resposta, o que demonstrou a motivação e o envolvimento no jogo. É importante, assim, ressaltar que houve, durante esta atividade, a contribuição para a construção do conhecimento, de forma que a maioria das perguntas foi respondida corretamente pelos alunos. 


\section{CONCLUSÃO}

A utilização de metodologias alternativas, pelo projeto de extensão, mostrouse positiva, por promover integração dos participantes, assim como por despertar o interesse e a motivação para o aprendizado das temáticas, oportunizando o desenvolvimento de habilidades que colaboraram na construção do conhecimento. Cabe-se, ainda, ressaltar que foi proporcionado aos profissionais de saúde, em formação, o contato com a comunidade, para a troca de experiências e conhecimento de sua realidade e seus anseios. A partir do que foi observado pelos extensionistas, constatou-se o déficit no entendimento de conteúdos básicos de Biologia e as dificuldades na comunicação com a família, sobre sexo/ sexualidade; e, ainda, a falta de diálogo a respeito da saúde sexual, no âmbito escolar.

Desse modo, ressalta-se a carência de ações promotoras de saúde, na educação da rede pública de ensino; e a necessidade de estratégias que visem à integração familiar, para discussão de temas importantes que explorem/abordem e foquem na saúde pública. Por fim, espera-se que este relato possa contribuir para discussões e reflexões futuras acerca do processo de ensino e aprendizagem, no que se refere às ações educativas em saúde, promovidas pelas Universidades públicas/instituições de Ensino Superior, relacionadas aos temas de grande relevância na atualidade, junto/direcionadas aos jovens e adolescentes, nos espaços escolares da Rede Estadual de Ensino. 


\section{REFERÊNCIAS BIBLIOGRÁFICAS}

ALMEIDA, Edmar Rocha, MOUTINHO, Cinara Botelho; LEITE, Maisa Tavares de Souza. Prática pedagógica de enfermeiros de Saúde da Família no desenvolvimento da Educação em Saúde. Interface - Comunicação, Saúde, Educação, v. 20, n. 57, p. 389-402, 2016.

BEZERRA, Sara Taciana Firmino et al. Promoción de la salud: la calidad de vida en las prácticas de enfermería. Enferm Global, v. 12, n. 32, p. 260-9, out. 2013. Disponível em: <https://revistas.um.es/eglobal/article/view/eglobal.12.4.172251>. Acesso em: 29 out. 2020.

CARTA DE OTTAWA. Saúde - Blog OPAS. Disponível em: <http://www.opas.org.br/coletiva/carta.cfm>. Acesso em: 26 jul. 2020.

DONALISIO, Maria Rita; FREITAS, André Ricardo Ribas; ZUBEN, Andrea Paula Bruno Von. Arboviroses emergentes no Brasil: desafios para a clínica e implicações para a saúde pública. Rev. Saúde Pública, São Paulo, v. 51, n. 30, 2017. Disponível em: $<$ http://www.scielo.br/scielo.php?script=sci_arttext\&pid=S0034-

$89102017000100606 \&$ Ing=en\&nrm=iso>. Acesso em: 25 jul. 2020.

FERREIRA, Marcos Santos; CASTIEL, Luis David; CARDOSO, Maria Helena Cabral de Almeida. Sedentarismo mata? Estudo dos comentários de leitores de um jornal brasileiro online. Saúde e Sociedade, São Paulo, v. 26, n. 1, p. 15-28, mar. 2017. Disponível em: $<$ http://www.scielo.br/scielo.php?script=sci_arttext\&pid=S010412902017000100015\&lng=en\&nr m=iso>. Acesso em: 29 ago. 2020.

FREIRE, Paulo. Pedagogia da autonomia: saberes necessários à prática educativa. 60 . ed. São Paulo: Paz e Terra, 2019.

MOURA, Denizielle de Jesus Moreira et al. Construção de cartilha sobre insulinoterapia para crianças com diabetes mellitus tipo 1. Rev. Bras. Enferm., Brasília, v. 70, n. 1, p. 7-14, fev. 2017. Disponível em: <http://www.scielo.br/scielo.php?script=sci_arttext\&pid=S003471672017000100007\&Ing=en\&nrm=iso>. Acesso em: 03 set. 2020.

SANTOS, Marcos Eduardo Miranda et al. Ações educativas para o combate ao mosquito Aedes Aegypti em uma escola da Região Metropolitana de São Luís. Revista Caderno Pedagógico, v. 14, n. 1, 2017.

SCHALET, A. T.; SANTELLI, J. S.; RUSSELL, S. T. et al. Invited Commentary: Broadening the Evidence for Adolescent Sexual and Reproductive Health and Education in the United States. Journal of Youth and Adolescence, v. 43, n. 10, p. 1595-1610, 2014.

SCULL, T. M.; MALIK, C. V.; MORRISON, A. et al. Study protocol for a randomized controlled trial to evaluate a web-based comprehensive sexual health and media literacy education program for high school students. Trials, v. 21, n. 1, 2020.

WORLD HEALTH ORGANIZATION. Health promotion evaluation: recommendations to policymakers. Report of the WHO European Working Group on Health Promotion Evaluation. Copenhagen: WHO, $1998 . \quad$ Disponível em: <https://apps. who.int/iris/bitstream/handle/10665/108116/E60706. pdf?sequence=1\&isAllowed=y> . Acesso em: 31 ago. 2020. 